\title{
Quantifying right ventricular diffuse fibrosis in tetralogy of Fallot - a novel customised approach for the challenges of the right ventricle
}

Ee Ling Heng ${ }^{1,2^{*}}$, Peter Kellman ${ }^{3}$, Michael A Gatzoulis ${ }^{1,2}$, James Moon ${ }^{4}$, Peter Gatehouse ${ }^{1,5}$, Sonya $\mathrm{V}$ Babu-Narayan ${ }^{1,2}$

From 19th Annual SCMR Scientific Sessions

Los Angeles, CA, USA. 27-30 January 2016

\section{Background}

There are clear clinical drivers for right ventricular (RV) T1 mapping in patients with repaired tetralogy of Fallot
(rTOF), in whom myocardial fibrosis is implicated in adverse clinical outcomes. However, considerable technical challenges exist, due to thin mobile RV wall with

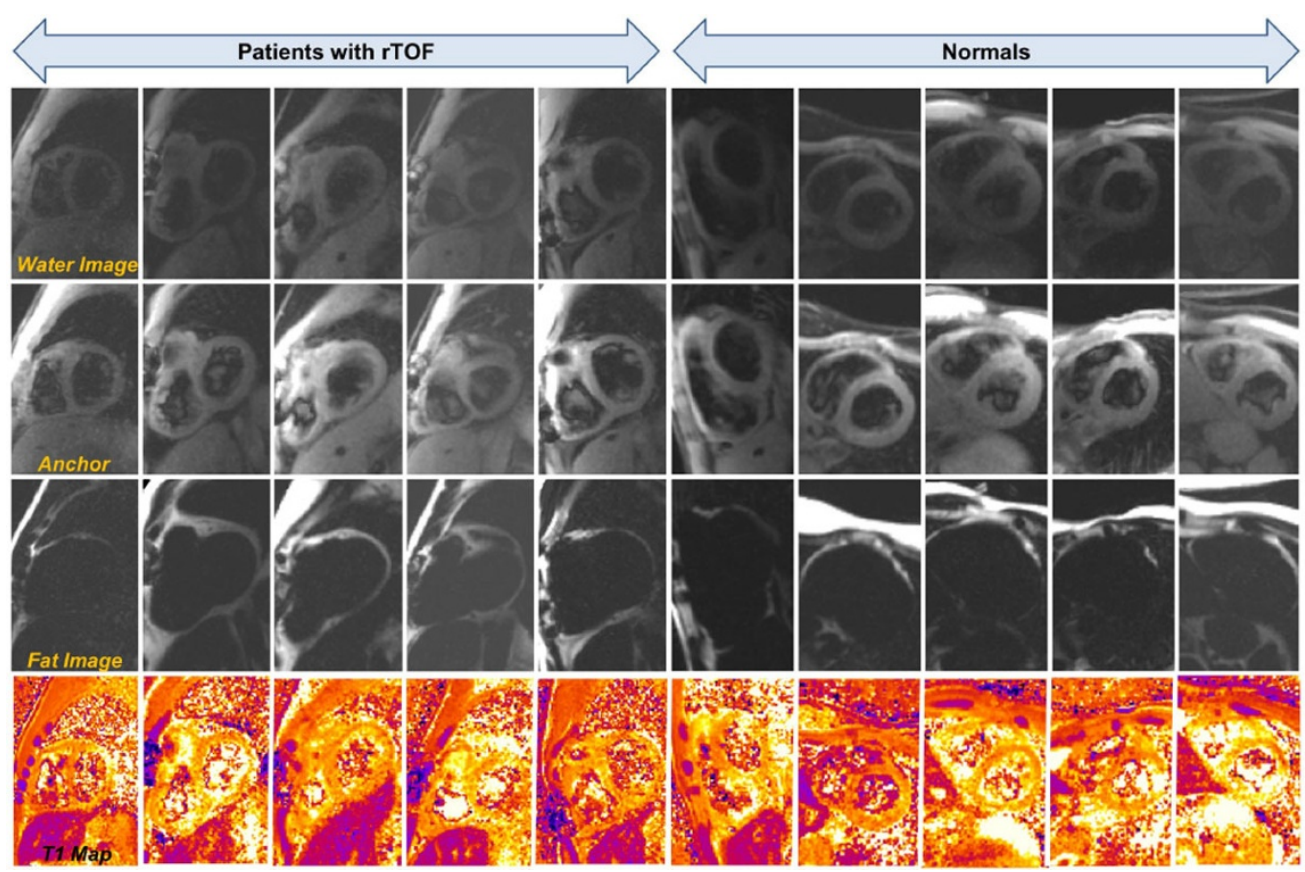

Figure 1 Representative short-axis images from subjects scanned for RV T1 mapping by fat-water separated, MSPrep dark blood imaging. Images for five subjects with repaired tetralogy of Fallot and five healthy volunteers shown (one subject per column): Top row - MoCo averaged water-only image at Ts 600 ms, Second row - MoCo averaged water-only anchor image at same window/level, Third row - MoCo averaged fat only image, Bottom row - T1 map generated from registration and 2-parameter fit of the six images per sampling scheme.

1NIHR Cardiovascular Biomedical Research Unit, Royal Brompton \& Harefield

NHS Foundation Trust and Imperial College London, London, UK

Full list of author information is available at the end of the article 


\begin{tabular}{|c|c|c|c|}
\hline & $\begin{array}{l}\text { rTOF } \\
(n=11)\end{array}$ & $\begin{array}{c}\text { Controls } \\
(n=11)\end{array}$ & $p$ value \\
\hline Age, years & $37.5 \pm 9.5$ & $35.3 \pm 8.9$ & 0.59 \\
\hline Gender (Male/Female) & $7 / 4$ & $6 / 5$ & 0.73 \\
\hline RVEDVi, $\mathrm{ml} / \mathrm{m}^{2}$ & $102.8 \pm 22.4$ & $75.6 \pm 16.3$ & 0.04 \\
\hline RVESVi, ml/m² & $49.2 \pm 14.0$ & $25.4 \pm 8.7$ & $<0.01$ \\
\hline RVSVi, ml/m² & $53.6 \pm 10.5$ & $50.4 \pm 9.7$ & 0.47 \\
\hline RVEF, \% & $52.6 \pm 5.1$ & $66.7 \pm 6.4$ & $<0.01$ \\
\hline RVMi, $g / m^{2}$ & $37.3 \pm 11.6$ & $30.3 \pm 6.0$ & 0.09 \\
\hline LVEDVi, $\mathrm{ml} / \mathrm{m}^{2}$ & $85.9 \pm 16.4$ & $76.4 \pm 13.5$ & 0.16 \\
\hline LVESVi, $\mathrm{ml} / \mathrm{m}^{2}$ & $35.8 \pm 10.0$ & $25.6 \pm 6.1$ & 0.01 \\
\hline LVSVi, ml/m² & $50.0 \pm 8.2$ & $50.8 \pm 8.6$ & 0.83 \\
\hline LVEF, \% & $58.8 \pm 5.2$ & $66.6 \pm 3.9$ & $<0.01$ \\
\hline LVMi, g/m² & $58.0 \pm 10.2$ & $59.9 \pm 7.4$ & 0.62 \\
\hline Pulmonary regurgitant fraction, $\%$ & $6.2 \pm 13.6$ & $0.7 \pm 1.2$ & 0.44 \\
\hline $\mathrm{RAAi}, \mathrm{cm}^{2} / \mathrm{m}^{2}$ & $13.7 \pm 5.4$ & $10.6 \pm 1.4$ & 0.08 \\
\hline LAAi, $\mathrm{cm}^{2} / \mathrm{m}^{2}$ & $9.8 \pm 3.7$ & $10.7 \pm 1.5$ & 0.49 \\
\hline \multicolumn{4}{|l|}{$\underline{\text { RV T1 }}$} \\
\hline Native RV myocardial T1, ms & $1233 \pm 52$ & $1270 \pm 97$ & 0.28 \\
\hline Post Gd RV myocardial T1, ms & $364 \pm 37$ & $341 \pm 34$ & 0.15 \\
\hline RV ECV, \% & $43.4 \pm 6.2$ & $44.5 \pm 7.0$ & 0.70 \\
\hline \multicolumn{4}{|l|}{ LV MOLLI } \\
\hline Native LV myocardial T1, ms & $1016 \pm 35$ & $1019 \pm 29$ & 0.85 \\
\hline Post Gd LV myocardial T1, ms & $461 \pm 38$ & $455 \pm 28$ & 0.67 \\
\hline Pre Gd blood T1,ms & $1600 \pm 60$ & $1631 \pm 82$ & 0.32 \\
\hline Post Gd blood T1, ms & $308 \pm 28$ & $289 \pm 26$ & 0.12 \\
\hline MOLLI LV ECV, \% & $26.3 \pm 3.2$ & $25.1 \pm 2.7$ & 0.33 \\
\hline
\end{tabular}

adjacent strong signals from blood and epicardial fat. We prospectively aimed to explore the possible clinical significance of RV diffuse fibrosis in rTOF compared to health.

\section{Methods}

Technical

We adopted a saturation recovery (SASHA) based approach with MoCo averaging of single-shot fat-water separated images at $1.5 \mathrm{~T}$ (Siemens Avanto, 32-channel thoracic phased-array). ${ }^{4}$ We applied Motion-sensitive dephasing (MSPrep) to null blood signal before each shot. ${ }^{1-3}$ Composite saturation and MSPrep parameters were optimised prior to four 10-cycle scans at saturation-recovery delay Ts $\approx 600 \mathrm{~ms}$ and two "anchor" scans at Ts $>6 \mathrm{sec}^{5}$ run free-breathing twice pre-contrast administration (Figure 1). Complex non-rigid MoCo averaging $^{6}$ (fixed 50\% acceptance) incorporated 5 shots at the most similar respiratory phase. Imaging parameters: $\mathrm{TE}=1.0,2.7,4.3 \mathrm{~ms}$ (reduced to tolerate proximity to sternal wires), FA $10^{\circ}, 6 \times 1.9 \times 2.1 \mathrm{~mm}$ acquired voxels, TGRAPPA rate 4 , diastolic shot 
duration $175 \mathrm{~ms}$. Post-contrast ( $0.15 \mathrm{mmol} / \mathrm{kg}$ Gadovist) RV T1 scans used Ts $\approx 200 \mathrm{~ms}$ and "anchor" Ts $>3 \mathrm{sec}$. Separate 11-HB MOLLIs were acquired for blood T1 and LV ECV, at a LV-aligned short-axis plane pre-contrast (5(3)3) and 15 minutes post-contrast (4(1)3(1)2).

\section{Clinical}

Data for 22 subjects (11 patients with rTOF and 11 age and gender-matched healthy volunteers) were acquired. Two-parameter fit pixelwise T1 maps were generated for each run. Mean RV free wall T1s were measured by two independent observers using CMR42. Interobserver reproducibility was calculated by coefficient of variation $(\mathrm{CoV} \%)=$ (within-subject standard deviation/mean) $\mathrm{x} 100 \%$.

\section{Results}

RV T1 maps were obtained in all subjects, with interobserver reproducibility of native RV myocardial T1 (CoV 1.8\%) and RV ECV (CoV 6.8\%). There was no significant difference in RV native T1 and ECV of patients with rTOF compared to the controls who had thin RV walls (Figure 2). This may reflect the modest sample size, or the inclusion of clinically stable patients with rTOF with minimal residual haemodynamic lesions, or may also reflect technical limitations. Saturation was optimised to $<1 \%$ in the RV but non-uniformity over the heart requires investigation. The MSPrep required subject-specific optimisation for minimal partial volume contamination by blood which may explain high RV ECV. Known underestimation of native T1 by MOLLI compared to SASHA may also explain some of the RV-LV difference.

\section{Conclusions}

RV T1 and ECV quantification is possible with the proposed technique but requires further development. The diagnostic value in this patient population merits further work towards a larger study and to explore histological correlation.

\footnotetext{
Authors' details

${ }^{1} \mathrm{NIHR}$ Cardiovascular Biomedical Research Unit, Royal Brompton \& Harefield NHS Foundation Trust and Imperial College London, London, UK. ${ }^{2}$ Department of Adult Congenital Heart Disease, Royal Brompton Hospital, London, UK. ${ }^{3}$ National Heart, Lung and Blood Institute, National Institutes of Health, Bethesda, MD, USA. ${ }^{4}$ Heart Hospital Imaging Centre, The Heart Hospital, UCLH \& UCL, London, UK. ${ }^{5}$ Cardiovascular Magnetic Resonance Unit, Royal Brompton Hospital, London, UK.
}

Published: 27 January 2016
doi:10.1186/1532-429X-18-S1-O26

Cite this article as: Heng et al:: Quantifying right ventricular diffuse fibrosis in tetralogy of Fallot - a novel customised approach for the challenges of the right ventricle. Journal of Cardiovascular Magnetic Resonance 2016 18(Suppl 1):026.

\section{Submit your next manuscript to BioMed Central and take full advantage of:}

- Convenient online submission

- Thorough peer review

- No space constraints or color figure charges

- Immediate publication on acceptance

- Inclusion in PubMed, CAS, Scopus and Google Scholar

- Research which is freely available for redistribution

Submit your manuscript at www.biomedcentral.com/submit 